\title{
The fundamental properties of time varying AR models with non stochastic coefficients
}

\author{
M. Karanasos ${ }^{\dagger}$, A. G. Paraskevopoulos and S. Dafnos \\ Brunel University, London, UK
}

This draft: March 8th 2014

\begin{abstract}
The paper examines the problem of representing the dynamics of low order autoregressive (AR) models with time varying (TV) coefficients. The existing literature computes the forecasts of the series from a recursion relation. Instead, we provide the linearly independent solutions to TV-AR models. Our solution formulas enable us to derive the fundamental properties of these processes, and obtain explicit expressions for the optimal predictors. We illustrate our methodology and results with a few classic examples amenable to time varying treatment, e.g, periodic, cyclical, and AR models subject to multiple structural breaks.

Keywords: abrupt breaks, covariance structure, cyclical processes, homogeneous and particular solutions, optimal predictors, periodic AR models.
\end{abstract}

We gratefully acknowledge the helpful conversations we had with L. Giraitis, G. Kapetanios and A. Magdalinos in the preparation of the paper. We would also like to thank R. Baillie, L. Bauwens, M. Brennan, D. van Dijk, W. Distaso, C. Francq, C. Gourieroux, E. Guerre, M. Guidolin, A. Harvey, C. Hommes, S. Leybourne, P. Minford, A. Monfort, C. Robotti, W. Semmler, R. Smith, T. Teräsvirta, P. Zaffaroni, and J-M Zakoian for suggestions and comments on a closely related work (see Paraskevopoulos et al., 2013) which greatly improved many aspects of the current paper as well. We are grateful to seminar participants at CREST, Erasmus University, London School of Economics, Queen Mary University of London, Imperial College, University of Essex, Birkbeck College University of London, University of Nottingham, Cardiff University, University of Manchester, Athens University of Economics and Business, and University of Piraeus. We have also benefited from the comments given by participants (on the closely related work) at the 3rd Humboldt-Copenhagen Conference on Financial Econometrics (Humboldt University, Berlin, March 2013), the SNDE 21st Annual Symposium (University of Milan-Bicocca, March 2013), the 8th and 9th BMRC-QASS conferences on Macro and Financial Economics (Brunel University, London, May 2013), the 7th CFE Conference (Senate House, University of London, December 2013), and the 1st RASTANEWS Conference (University of Milan-Bicocca, January 2014).

${ }^{\dagger}$ Address for correspondence: Menelaos Karanasos, Economics and Finance, Brunel University, West London, UB3 3PH, UK; email: menelaos.karanasos@brunel.ac.uk, tel: +44(0)1895265284, fax: +44 (0)1895269770. 


\section{Introduction}

The constancy of the parameters assumption made in the specification of time series econometric models has been the subject of criticism for a long time. It is argued that the assumption is inappropriate in the face of changing institutions and a dynamically responding economic policy. These evolving factors cause the parameter values characterizing economic relationships to change over time. Partly to respond to the criticism and partly motivated by the desire to construct dynamic models, econometricians have developed an arsenal of powerful methods that attempt to capture the evolving nature of our economy. Such frameworks include AR processes which contain multiple abrupt breaks, and periodic and cyclical autoregressive models.

A methodology is presented in this paper for analyzing time varying systems which is also applicable to the three aforementioned processes. A technique is set forth for examining the periodic AR model, which overcomes the usual requirement of expressing the periodic process in a vector AR (VAR) form.

The first attempts to develop theories for time varying models, made in the 1960's, were based on a recursive approach (Whittle, 1965) and on evolutionary spectral representations (Abdrabbo and Priestley, 1967). Rao (1970) used the method of weighted least squares to estimate an autoregressive model with time dependent coefficients. Despite nearly half a century of research work, the great advances, and the widely recognized importance of time varying structures, the bulk of econometric models have constant coefficients. There is a lack of a general theory that can be employed to systematically explore their time series properties. Granger in some of his last contributions highlighted the importance of the topic (see, Granger 2007, and 2008).

There is a general agreement that the main obstacle to progress is the lack of a universally applicable method yielding a closed form solution to stochastic time varying difference equations. The present paper is part of a research program aiming to produce and utilize closed form solutions to AR processes with non stochastic time dependent coefficients. Our methodology attempts to trace the path of these changing coefficients. To be specific, in the time series literature, there is no method for finding the $p$ linearly independent solutions that we need to obtain the general solution of the TV-AR model of order $p$. To keep the exposition tractable and reveal its practical significance we work with low order specifications.

The main part of the paper begins with subsection 2.2 , where we state the second order difference equation with time variable coefficients, which is our main object of inquiry. We start by writing this equation in a more efficient way as an infinite linear system. The next step is to define the matrix of coefficients, called the fundamental solution matrix, associated with the system representation. This 
matrix is a workhorse of our research and it is derived step by step from the time varying coefficients of the difference equation.

The reader will have noticed that we have moved the goalposts, paradoxically against us, from obtaining a solution for a time varying (low order) difference equation, to solving an infinite linear system. The reason is that the solution of such infinite systems has been made possible recently, due to an extension of the standard Gaussian elimination, called the infinite Gaussian elimination (see Paraskevopoulos, 2012). Applying this infinite extension algorithm, we obtain the fundamental solutions, which take explicit forms in terms of the determinants of the fundamental solution matrix.

Subsection 2.3 contains the main theoretical result of the paper. Pursuing the conventional route followed by the differential and difference equations literature, we construct the general solution by finding its two parts, the homogeneous one and a particular part. It is expressed as Theorem 1 and its proof is in Appendix A. The coefficients in these solutions are expressed as determinants of tridiagonal matrices. The second order properties of the TV-AR process can easily be deduced from these solutions. An additional benefit of these solutions is the facility with which linear prediction can be produced. This allows us to provide a thorough description of time varying models by deriving: first, multistep ahead forecasts, the associated forecast error and the mean square error; second, the first two unconditional moments of the process and its covariance structure. In related works we provide results for the $p$ order and the more general ascending order (see, for example, Paraskevopoulos et al., 2013). Our method is a natural extension of the first order solution formula. It also includes the linear difference equation with constant coefficients (see, for example, Karanasos, 2001) as a special case.

The next two Sections of the paper, 3 and 4, apply our theoretical framework to a few classic time series models, which are obvious candidates for a time varying treatment. Linear systems with time dependent coefficients are not only of interest in their own right, but, because of their connection with periodic models and time series data which are subject to structural breaks. They also provide insight into these processes as well. Viewing a periodic AR (PAR) formulation as a TV model clearly obviates the need for VAR analysis. For surveys and a review of some important aspects of PAR processes see Franses (1996b), Franses and Paap (2004), Ghysels and Osborn (2001), and Hurd and Miamee (2007). The authoritative studies by Osborn (1988), Birchenhall et al. (1989), and Osborn and Smith (1989) applied these models to consumption. del Barrio Castro and Osborn (2008) pointed out that "despite the attraction of PAR models from the perspective of economic decision making in a seasonal context, the more prominent approach of empirical workers is to assume that the AR coefficients, except for the intercept, are constant over the seasons of the year" 1

\footnotetext{
${ }^{1}$ del Barrio Castro and Osborn $(2008,2012)$ (see the references therein for this stream of important research; see also
} 
Despite the recognized importance of periodic processes for economics there have been few attempts to investigate their time series properties (see, among others, Franses, 1994, Franses, 1996a, Lund and Basawa, 2000, Franses and Paap, 2005). Tiao and Grupe (1980) and Osborn (1991) analyzed these models by converting them into a VAR process with constant coefficients. In this paper we develop a general theory that can be employed to systematically explore the fundamental properties of the periodic formulation. We remain within the univariate framework and we look upon the PAR model as a stochastic difference equation with time varying (albeit periodically varying) parameters.

Although some theoretical analysis of periodic specifications was carried out by the aforementioned studies the investigation of their fundamental properties appears to have been limited to date. Cipra and Tlustý (1987), Anderson and Vecchia (1993), Adams and Goodwin (1995), Shao (2008), and Tesfaye et al. (2011) discuss parameter estimation and asymptotic properties of periodic AR moving average (PARMA) specifications. Bentarzi and Hallin (1994) and McLeod (1994) derive invertibility conditions and diagnostic checks for such processes. Lund and Basawa (2000) develop a recursive scheme for computing one-step ahead predictors for PARMA specifications, and compute multi-step-ahead predictors recursively from the one-step-ahead predictions. Anderson et al. (2013) develop a recursive forecasting algorithm for periodic models. We derive explicit formulas that allow the analytic calculation of the multi-step-ahead predictors.

We begin Section 3 with a $\operatorname{PAR}(2)$ model. We limit our analysis to a low order to save space and also since Franses (1996a) has documented that low order PAR specifications often emerge in practice. First, we formulate it as a TV model; then, we express its fundamental solution matrix as a block Toeplitz matrix. This representation enables us to establish an explicit formula for the general solution in terms of the determinant of such a block matrix. The result is presented in Proposition 1 which is the equivalent to Theorem 1 with the incorporation of the seasonal effects. That is, by taking account of seasons and periodicities, we obtain the general solution, by constructing its homogeneous and particular parts and then adding them up. In subsection 3.1, we turn our attention to a different type of seasonality, namely the cyclical AR (CAR) model and we provide its solution.

Section 4 is an application of the time varying framework to time series subject to multiple structural breaks. We employ a technique analogous to the one used in Section 3 on the PAR formulation. In particular, we express the fundamental solutions of the $\operatorname{AR}(2)$ model with $r$ abrupt breaks, as determinants of block tridiagonal matrices. Again, we are able to obtain the general solution by finding and adding the homogeneous and particular solutions.

One of the advantages of our time varying framework is that we can trace the entire path of the Taylor, 2002, 2003 and 2005) test for seasonal unit roots in integrated PAR models. 
series under consideration. In Section 5, we employ this information feature to derive the fundamental properties of the various TV-AR processes. For example, simplified closed-form expressions of the multistep forecast error variances are derived for time series when low order PAR models adequately describe the data. These formulae allow a fast computation of the multi-step-ahead predictors. Finally, Section 6 concludes.

\section{Time Varying AR Models}

\subsection{Preliminaries and Purpose of Analysis}

\subsubsection{Notation}

Throughout the paper we adhere to the following conventions: $\left(\mathbb{Z}^{+}\right) \mathbb{Z}$, and $\left(\mathbb{R}^{+}\right) \mathbb{R}$ stand for the sets of (positive) integers, and (positive) real numbers, respectively. Matrices and vectors are denoted by upper and lower case boldface symbols, respectively. For square matrices $\mathbf{X}=\left[x_{i j}\right]_{i, j=1, \ldots, k} \in \mathbb{R}^{k x k}$ using standard notation, $\operatorname{det}(\mathbf{X})$ or $|\mathbf{X}|$ denotes the determinant of matrix $\mathbf{X}$ and $\operatorname{adj}(\mathbf{X})$ its adjoint matrix. To simplify our exposition we also introduce the following notation: $t \in \mathbb{Z},(n, l) \in \mathbb{Z}^{+} \times \mathbb{Z}^{+} ; T=0, \ldots, n$ denotes the 'periods' (i.e., years); $s=1, \ldots, l$, denotes the 'seasons' (i.e, quarters in a year: $l=4$ ). The $t$ represents the present time and $k \in \mathbb{Z}^{+}$the number of seasons such that at time $\tau_{k}=t-k$ information is given.

Let the triple $\left(\Omega,\left\{F_{t}, t \in \mathbb{Z}\right\}, P\right)$ denote a complete probability space with a filtration, $\left\{F_{t}\right\}$, which is a non-decreasing sequence of $\sigma$-fields $F_{t-1} \subseteq F_{t} \subseteq F, t \in \mathbb{Z}$. The space of $P$-equivalence classes of finite complex random variables with finite $p$-order is indicated by $L_{p}$. Finally, $H=L_{2}\left(\Omega, F_{t}, P\right)$ stands for a Hilbert space of random variables with finite first and second moments.

\subsubsection{The Problem}

The solution of the second order linear difference equation with non constant coefficients is the building block for the extension of the well known closed form solution of the first order to the $p$ th order time varying equation. As noted by Sydsaeter et al. (2008), in their classic text (Further Mathematics for Economic Analysis, p. 403), in the case of second order homogeneous linear difference equations with variable coefficients:

"There is no universally applicable method of discovering the two linearly independent solutions that we need in order to find the general solution of the equation."

We can identify two lines of inquiry that can be pursued to solve linear difference equations with time 
varying coefficients. Searching for a solution, one can follow either of the following two paths. The first is to develop an analogous method to the standard one that exists for the linear $p$ order difference equation with constant coefficients: find the eigenvalues, solve the characteristic equation, and obtain the closed form. The second line of research searches for the generalization of the closed form formula that exists for first order time varying difference equations. Here, the way to proceed is to make up a conjecture and try to prove it by induction. The two strands of the literature have taken important steps, but have not provided us with a general solution method that we can apply; the existing results lack generality and applicability. To be more specific, the research problem we face is that there is a lack of a universally applicable method yielding a closed form solution to stochastic higher order difference equations with time dependent coefficients.

A general method for solving infinite linear systems with row-finite coefficient matrices has recently been established by Paraskevopoulos (2012). It is a modified version of the standard Gauss-Jordan elimination method implemented under a right pivot strategy, called infinite Gauss-Jordan elimination. Expressing the linear difference equation of second order with time dependent coefficients as an infinite linear system, the Gaussian elimination part of the method is directly applicable. It generates two linearly independent homogeneous solution sequences. The general term of each solution sequence turns out to be a continuant determinant. The general solutions of the homogeneous and nonhomogeneous difference equation are expressible as a single Hessenbergian, that is, a determinant of a lower Hessenberg matrix (see Karanasos, Paraskevopoulos and Dafnos 2013). Theorem 3 in Paraskevopoulos et al. (2013) affords an easy means of finding, for a given lower Hessenberg matrix, its ordinary expansion in non-determinant form (see also Paraskevopoulos and Karanasos, 2013). These results are extendible to the solution of the $p$ th and ascending order time varying linear difference equations in terms of a single Hessenbergian (see Paraskevopoulos et al., 2013). This makes it possible to introduce, in the above cited reference, a unified theory for time varying models.

\subsection{Fundamental Solution Matrices}

The main theoretical contribution of this Section is the development of a method that provides the closed form of the general solution to a TV-AR(2) model.

Next we give the main definition that we will use in the rest of the paper. Consider a second order stochastic difference equation with time dependent coefficients, which is equivalent to the time varying $\mathrm{AR}(2)$ process, given by

$$
y_{t}=\phi_{0}(t)+\phi_{1}(t) y_{t-1}+\phi_{2}(t) y_{t-2}+\varepsilon_{t}
$$


where $\left\{\varepsilon_{t}, t \in \mathbb{Z}\right\}$ is a sequence of zero mean serially uncorrelated random variables defined on $L_{2}\left(\Omega, F_{t}, P\right)$ with $\mathbb{E}\left[\varepsilon_{t} \mid F_{t-1}\right]=0$ a.s., and finite variance: $0<M_{l}<\sigma_{t}^{2}<M<\infty, \forall t,\left(M_{l}, M\right) \in \mathbb{R}^{+} \times \mathbb{R}^{+}$.

Remark 1 We have relaxed the assumption of homoscedasticity (see also, among others, Paraskevopoulos et al., 2013 and Karanasos et al., 2013), which is likely to be violated in practice and allow $\varepsilon_{t}$ to follow, for example, a periodical GARCH type of process (see, Bollerslev and Ghysels, 1996).

The fundamental solution sequence, and in general all the solution sequences, must necessarily be functions of the independent variable $t$, so as to satisfy eq. (1). Our intermediate objective is to obtain the fundamental solution matrix, denoted below by $\boldsymbol{\Phi}_{t, k}$, which is associated with our stochastic difference equation (1); the $\boldsymbol{\Phi}_{t, k}$ matrix will be derived from the time varying coefficients of eq. (11). The best way to appreciate the representation of the fundamental solution matrix is to view the stochastic difference equation as a linear system. We carry out this construction below. Once we have this stepping stone in place, then we can pursue our ultimate objective, by computing the determinants of the $\boldsymbol{\Phi}_{t, k}$, which will give us the linearly independent solutions sequences to the difference equation.

Equation (1) written as

$$
\phi_{2}(t) y_{t-2}+\phi_{1}(t) y_{t-1}-y_{t}=-\left[\phi_{0}(t)+\varepsilon_{t}\right]
$$

takes the infinite row (and column)-finite system form

$$
\Phi \cdot \mathbf{y}=-\phi-\varepsilon
$$

where

$$
\boldsymbol{\Phi}=\left(\begin{array}{ccccccc}
\phi_{2}\left(\tau_{k}+1\right) & \phi_{1}\left(\tau_{k}+1\right) & -1 & 0 & 0 & 0 & \ldots \\
0 & \phi_{2}\left(\tau_{k}+2\right) & \phi_{1}\left(\tau_{k}+2\right) & -1 & 0 & 0 & \ldots \\
0 & 0 & \phi_{2}\left(\tau_{k}+3\right) & \phi_{1}\left(\tau_{k}+3\right) & -1 & 0 & \ldots \\
\vdots & \vdots & \vdots & \vdots & \vdots & \vdots & \ldots
\end{array}\right)
$$


(row-finite is an infinite matrix whose rows have finite non zero elements) and

$$
\mathbf{y}=\left(\begin{array}{l}
y_{\tau_{k}-1} \\
y_{\tau_{k}} \\
y_{\tau_{k}+1} \\
y_{\tau_{n}+2} \\
y_{\tau_{k}+3} \\
y_{\tau_{k}+4} \\
\vdots
\end{array}\right), \boldsymbol{\phi}=\left(\begin{array}{c}
\phi_{0}\left(\tau_{k}+1\right) \\
\phi_{0}\left(\tau_{k}+2\right) \\
\phi_{0}\left(\tau_{k}+3\right) \\
\vdots
\end{array}\right), \boldsymbol{\varepsilon}=\left(\begin{array}{c}
\varepsilon_{\tau_{k}+1} \\
\varepsilon_{\tau_{k}+2} \\
\varepsilon_{\tau_{k}+3} \\
\vdots
\end{array}\right)
$$

(recall that $\tau_{k}=t-k$ ). The system representation results from the values that the coefficients take in successive time periods. The equivalence of (2) and (3) follows from the fact that the $i$ th equation in (3) , as a result of the multiplication of the $i$ th row of $\boldsymbol{\Phi}$ by the column of $y$ s equated to $-\left[\phi_{0}\left(\tau_{k}+i\right)+\varepsilon_{\tau_{k}+i}\right]$, is equivalent to eq. (2), as of time $\tau_{k}+i$. The $\boldsymbol{\Phi}$ matrix in eq. (3) can be partitioned as

$$
\mathbf{\Phi}=\left(\begin{array}{l|l}
\mathbf{P} & \mathbf{C}
\end{array}\right),
$$

where

$$
\mathbf{P}=\left(\begin{array}{cc}
\phi_{2}\left(\tau_{k}+1\right) & \phi_{1}\left(\tau_{k}+1\right) \\
0 & \phi_{2}\left(\tau_{k}+2\right) \\
0 & 0 \\
\vdots & \vdots
\end{array}\right), \mathbf{C}=\left(\begin{array}{ccccc}
-1 & 0 & 0 & 0 & \ldots \\
\phi_{1}\left(\tau_{k}+2\right) & -1 & 0 & 0 & \ldots \\
\phi_{2}\left(\tau_{k}+3\right) & \phi_{1}\left(\tau_{k}+3\right) & -1 & 0 & \ldots \\
\vdots & \vdots & \vdots & \vdots & \ldots
\end{array}\right)
$$

That is, $\mathbf{P}$ consists of the first 2 columns of $\boldsymbol{\Phi}$ and the $j$ th column of $\mathbf{C}, j=1,2, \ldots$, is the $(2+j)$ th column of $\boldsymbol{\Phi}$. We will denote the 2 nd column of the $k \times 2$ top submatrix of the matrix $\mathbf{P}$ by $\phi_{t, k}$ :

$$
\left(\phi_{t, k}\right)^{\prime}=\left(\phi_{1}\left(\tau_{k}+1\right), \quad \phi_{2}\left(\tau_{k}+2\right), \quad 0, \quad \ldots, 0\right)
$$


The $k \times(k-1)$ top submatrix of matrix $\mathbf{C}$ is called the core solution matrix and is denoted as

$$
\mathbf{C}_{t, k}=\left(\begin{array}{ccccc}
-1 & & & & \\
\phi_{1}\left(\tau_{k}+2\right) & -1 & & & \\
\phi_{2}\left(\tau_{k}+3\right) & \phi_{1}\left(\tau_{k}+3\right) & -1 & & \\
& \ddots & \ddots & \ddots & \\
& & \phi_{2}(t-1) & \phi_{1}(t-1) & -1 \\
& & & \phi_{2}(t) & \phi_{1}(t)
\end{array}\right)
$$

(here and in what follows empty spaces in a matrix have to be replaced by zeros). The fundamental solution matrix is obtained from the core solution matrix $\mathbf{C}_{t, k}$, augmented on the left by the $\phi_{t, k}$ column. That is,

$$
\begin{aligned}
\boldsymbol{\Phi}_{t, k}= & \left(\phi_{t, k} \mid \mathbf{C}_{t, k}\right)= \\
& \left(\begin{array}{cccccc}
\phi_{1}\left(\tau_{k}+1\right) & -1 & & & & \\
\phi_{2}\left(\tau_{k}+2\right) & \phi_{1}\left(\tau_{k}+2\right) & -1 & & & \\
& \phi_{2}\left(\tau_{k}+3\right) & \phi_{1}\left(\tau_{k}+3\right) & -1 & & \\
& & \ddots & \ddots & \ddots & \\
& & & \phi_{2}(t-1) & \phi_{1}(t-1) & -1 \\
& & & & \phi_{2}(t) & \phi_{1}(t)
\end{array}\right),
\end{aligned}
$$

(recall that $\tau_{k}=t-k$ ). Formally $\boldsymbol{\Phi}_{t, k}$ is a square $k \times k$ matrix whose $(i, j)$ entry $1 \leq i, j \leq k$ is given by

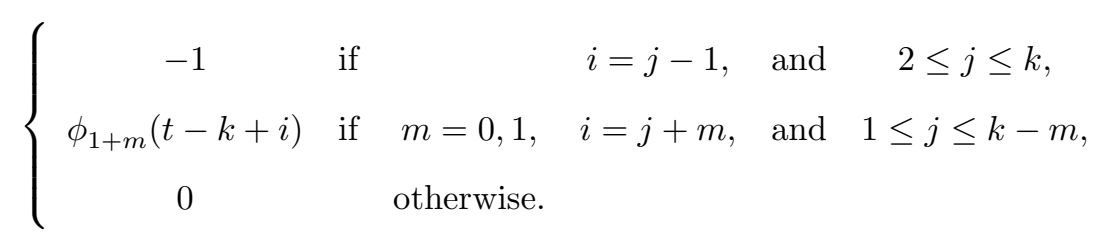

It is a continuant or tridiagonal matrix, that is a matrix that is both an upper and lower Hessenberg matrix. We may also characterize it as a 'time varying' Toeplitz matrix, because its time invariant version is a Toeplitz matrix of bandwidth 3 . We next define the bivariate function $\xi: \mathbb{Z} \times \mathbb{Z}^{+} \longmapsto \mathbb{R}$ by

$$
\xi_{t, k}=\operatorname{det}\left(\boldsymbol{\Phi}_{t, k}\right)
$$

coupled with the initial values $\xi_{t, 0}=1$, and $\xi_{t,-1}=0$. That is, $\xi_{t, k}$ for $k \geq 2$, is a determinant of a $k \times k$ 
matrix; each of the two nonzero diagonals (below the superdiagonal) of this matrix consists of the time varying coefficients $\phi_{m}(\cdot), m=1,2$, from $t-k+m$ to $t$. In other words, $\xi_{t, k}$ is a $k$ th-order tridiagonal determinant. Paraskevopoulos and Karanasos (2013) give its ordinary expansion in non-determinant form (a closed form solution).

\subsection{Main Theorem}

This short section contains the statement of our main theorem.

Theorem 1 The general solution of eq. (1) with free constants (initial condition values) $y_{t-k}, y_{t-k-1}$ is given by

$$
y_{t, k}^{g e n}=y_{t, k}^{h o m}+y_{t, k}^{p a r},
$$

where

$$
\begin{aligned}
y_{t, k}^{\text {hom }} & =\xi_{t, k} y_{t-k}+\phi_{2}(t-k+1) \xi_{t, k-1} y_{t-k-1}, \\
y_{t, k}^{\text {par }} & =\sum_{i=0}^{k-1} \xi_{t, i} \phi_{0}(t-i)+\sum_{i=0}^{k-1} \xi_{t, i} \varepsilon_{t-i} .
\end{aligned}
$$

In the above Theorem $y_{t, k}^{g e n}$ is decomposed into two parts: first, the $y_{t, k}^{h o m}$ part, which is written in terms of the two free constants $\left(y_{t-k-m}, m=0,1\right)$, and, second, the $y_{t, k}^{p a r}$ part, which contains the time varying drift terms and the error terms from time $t-k+1$ to time $t$.

Notice that the 'coefficients' of eq. (7), that is, the $\xi$ 's are expressed as continuant determinants. Moreover, for ' $k=0$ ' (for $i>j$ we use the convention $\sum_{q=i}^{j}(\cdot)=0$ ), since $\xi_{t, 0}=1$ and $\xi_{t,-1}=0$ (see eq. (6)), eq. (7) becomes an 'identity': $y_{t, 0}^{g e n}=y_{t}$. Similarly, when ' $k=1$ ' eq. (7), since $\xi_{t, 1}=\phi_{1}(t)$ and $\xi_{t, 0}=1$, reduces to $y_{t, 1}^{g e n}=\phi_{1}(t) y_{t-1}+\phi_{2}(t) y_{t-2}+\phi_{0}(t)+\epsilon_{t}$.

In the next Section, we illustrate the above claims in the context of a simple seasonal process with fixed periodicity, and a cyclical model as well.

\section{Periodic AR(2) Model}

Periodic regularities are phenomena occurring at the same season every year, so analogous to each other that we can view them as recurrences of the same event. Many economic time series are periodic in this sense. In the present Section we express them in a mathematical model, so that we can then employ it for forecasting and control. Gladyshev (1961) introduced a technique which still dominates the literature. He begins by decomposing the series into subperiods; then he treats each point within a subperiod as one 
part of a multivariate process. In this way he transforms a univariate non-stationary formulation into a multivariate stationary one. Following Gladyshev, Tiao and Grupe (1980) and Osborn (1991) treated periodic autoregressions as conventional nonperiodic VAR processes. But, as pointed out by Lund et al. (2006), even low order specifications can have an inordinately large numbers of parameters. A PAR(1) model for daily data, for example, has 365 autoregressive parameters. Its time invariant VAR form will contain 365 variables, and this is a handicap, especially for forecasting.

The most common case is the modeling in one dimensional time repetition at equal intervals. In this Section we present a re-examination of the periodic modeling problem. Our approach differs from most of the existing literature in that we stay within the univariate framework.

A periodic AR model of order 2 with $l$ seasons, $\operatorname{PAR}(2 ; l)$, is defined as

$$
y_{t_{s}}=\phi_{0, s}+\phi_{1, s} y_{t_{s}-1}+\phi_{2, s} y_{t_{s}-2}+\varepsilon_{t_{s}}
$$

where $t_{s}=T l+s, s=1, \ldots, l$, that is time $t_{s}$ is at the $s$ th season and $\phi_{m, s}, m=1,2$, are the periodically (or seasonally) varying autoregressive coefficients. For example, if $s=l$ (that is, we are at the $l$ th season) then the periodically varying coefficients are $\phi_{m, l}$ whereas if $s=1$ (that is, we are at the 1 st season) then the periodically varying coefficients are $\phi_{m, 1} ; \phi_{0, s}$ is a periodically varying drift. The above process nests the $\mathrm{AR}(2)$ model as a special case if we assume that the drift and all the AR parameters are constant, that is: $\phi_{m, s}=\varphi_{m}, m=0,1,2$, for all $t$.

The $\operatorname{PAR}(2 ; l)$ model can be expressed as the time varying $\operatorname{AR}(2)$ model in eq.(1):

$$
y_{t}=\phi_{0}(t)+\phi_{1}(t) y_{t-1}+\phi_{2}(t) y_{t-2}+\varepsilon_{t},
$$

where $\phi_{m}(t)=\phi_{m}\left(\tau_{T l}\right), m=0,1,2, \tau_{T l}=t-T l$, are the periodically (or seasonally) varying autoregressive coefficients: $\phi_{m, s} \triangleq \phi_{m}(T l+s), s=1, \ldots, l$.

For the $\operatorname{PAR}(2 ; l)$ model the continuant matrix $\boldsymbol{\Phi}_{t, n l}$ in eq. (5) (we assume that information is given at time $\tau_{n l}=t-n l$ for ease of exposition; it can of course be given at any time $\tau_{n l+s}=t-n l-s$ ) can be expressed as a block Toeplitz matrix. Thus, we have

$$
\xi_{t, n l}=\left|\boldsymbol{\Phi}_{t, n l}\right|,
$$


with

$$
\boldsymbol{\Phi}_{t, n l}=\left(\begin{array}{lllll}
\boldsymbol{\Phi}_{\tau_{(n-1) l}, l} & \overline{\mathbf{0}} & & & \\
\widetilde{\mathbf{0}}_{\tau_{n-2}} & \boldsymbol{\Phi}_{\tau_{(n-2) l}, l} & \overline{\mathbf{0}} & & \\
& \ddots & \ddots & \ddots & \\
& & \widetilde{\mathbf{0}}_{\tau_{1}} & \boldsymbol{\Phi}_{\tau_{l}, l} & \overline{\mathbf{0}} \\
& & & \widetilde{\mathbf{0}}_{t} & \boldsymbol{\Phi}_{t, l}
\end{array}\right),
$$

where $\overline{\mathbf{0}}$ is an $l \times l$ matrix of zeros except for -1 in its $(l, 1)$ entry; $\widetilde{\mathbf{0}}_{t}$ is an $l \times l$ matrix of zeros except $\phi_{2}(t-l+1)$, in its $(1, l)$ entry. Since $\phi_{m}\left(\tau_{T l}\right)=\phi_{m}(t): \widetilde{\mathbf{0}}_{\tau_{T l}}=\widetilde{\mathbf{0}}_{t}$ and $\boldsymbol{\Phi}_{\tau_{T l}, l}=\boldsymbol{\Phi}_{t, l}$. Thus the block diagonal matrix $\boldsymbol{\Phi}_{t, n l}$ can be written as

$$
\boldsymbol{\Phi}_{t, n l}=\left(\begin{array}{ccccc}
\boldsymbol{\Phi}_{t, l} & \overline{\mathbf{0}} & & & \\
\widetilde{\mathbf{0}}_{t} & \boldsymbol{\Phi}_{t, l} & \overline{\mathbf{0}} & & \\
& \ddots & \ddots & \ddots & \\
& & \widetilde{\mathbf{0}}_{t} & \boldsymbol{\Phi}_{t, l} & \overline{\mathbf{0}} \\
& & & \widetilde{\mathbf{0}}_{t} & \boldsymbol{\Phi}_{t, l}
\end{array}\right)
$$

where $\boldsymbol{\Phi}_{t, l}$ is the continuant or tridiagonal matrix $\boldsymbol{\Phi}_{t, k}$ matrix defined in eq. (5) when $k=l$. That is

$$
\boldsymbol{\Phi}_{t, l}=\left(\begin{array}{cccccc}
\phi_{1}\left(\tau_{l}+1\right) & -1 & & & & \\
\phi_{2}\left(\tau_{l}+2\right) & \phi_{1}\left(\tau_{l}+2\right) & -1 & & & \\
& \phi_{2}\left(\tau_{l}+3\right) & \phi_{1}\left(\tau_{l}+3\right) & -1 & & \\
& & \ddots & \ddots & \ddots & \\
& & & \phi_{2}(t-1) & \phi_{1}(t-1) & -1 \\
& & & & \phi_{2}(t) & \phi_{1}(t)
\end{array}\right)
$$

Proposition 1 The general solution of eq. (8) with free constants (initial condition values) $y_{t-n l}$, $y_{t-n l-1}$ is given by

$$
y_{t, n l}^{g e n}=y_{t, n l}^{h o m}+y_{t, n l}^{p a r}
$$

where

$$
\begin{aligned}
y_{t, n l}^{h o m} & =\xi_{t, n l} y_{t-n l}+\phi_{2}(t-n l+1) \xi_{t, n l-1} y_{t-n l-1}, \\
y_{t, n l}^{p a r} & =\sum_{s=0}^{l-1} \sum_{T=0}^{n-1} \xi_{t, T l+s} \phi_{0}(t-s)+\sum_{i=0}^{n l-1} \xi_{t, i} \varepsilon_{t-i},
\end{aligned}
$$


and $\xi_{t, n l}$ is given either in eq. (9) or in Proposition (6) in Appendix B.

The proof of the above Proposition follows immediately from Theorem 1 and the definition of the periodic model (8) .

\subsection{Cyclical AR(2) process}

Some economic series exhibit oscillations which are not associated with the same fixed period every year. Despite their lack of fixed periodicity, such time series are predictable to a certain degree.

Rather than setting up a general model from first principles, we re-interpret the periodic model with some modifications. In particular, we now assume that we have $d+1$ cycles, with $0 \leq d \leq l-1$. Then, $s_{j}=l_{j-1}+1, \ldots, l_{j}, j=1, \ldots, d+1$ (with $\left.0=l_{0}<l_{1}<\ldots<l_{d}<l_{d+1}=l\right)$ are the seasons in cycle $j$. Thus we can write $\phi_{m, s_{j}} \triangleq \phi_{m}\left(t_{s_{j}}\right), m=0,1,2, t_{s_{j}}=T l+s_{j}$. A CAR $(2)$ model with $l$ seasons and $d+1$ cycles $(\operatorname{CAR}(2 ; l ; d))$ is defined as

$$
y_{t_{j}}=\phi_{0, s_{j}}+\phi_{1, s_{j}} y_{t_{s_{j}}-1}+\phi_{2, s_{j}} y_{t_{s_{j}}-2}+\varepsilon_{t_{s_{j}}}
$$

For the above process, $\boldsymbol{\Phi}_{t, l}$ in eq. (11) can be written as

$$
\boldsymbol{\Phi}_{t, l}=\left[\begin{array}{lllll}
\boldsymbol{\Phi}_{t-l_{d}, l_{d+1}-l_{d}} & \overline{\mathbf{0}}_{d} & & & \\
\widetilde{\mathbf{0}}_{d} & \boldsymbol{\Phi}_{t-l_{d-1}, l_{d}-l_{d-1}} & \overline{\mathbf{0}}_{d-1} & & \\
& \ddots & \ddots & \ddots & \\
& & \widetilde{\mathbf{0}}_{2} & \boldsymbol{\Phi}_{t-l_{1}, l_{2}-l_{1}} & \overline{\mathbf{0}}_{1} \\
& & & \widetilde{\mathbf{0}}_{1} & \boldsymbol{\Phi}_{t, l_{1}}
\end{array}\right],
$$

where first, the $j(j=1, \ldots, d+1)$ block of the main diagonal is $\boldsymbol{\Phi}_{t-l_{j-1}, l_{j}-l_{j-1}}$, that is a $\left(l_{j}-l_{j-1}\right) \times$ $\left(l_{j}-l_{j-1}\right)$ banded 'time varying' Toeplitz matrix of bandwidth 3 :

$\boldsymbol{\Phi}_{t-l_{j-1}, l_{j}-l_{j-1}}=\left(\begin{array}{cccccc}\phi_{1}\left(\tau_{l_{j}}+1\right) & -1 & & & & \\ \phi_{2}\left(\tau_{l_{j}}+2\right) & \phi_{1}\left(\tau_{l_{j}}+2\right) & -1 & & & \\ & \phi_{2}\left(\tau_{l_{j}}+3\right) & \phi_{1}\left(\tau_{l_{j}}+3\right) & -1 & & \\ & & \ddots & \ddots & \ddots & \\ & & & \phi_{2}\left(\tau_{l_{j-1}}-1\right) & \phi_{1}\left(\tau_{l_{j-1}}-1\right) & -1 \\ & & & \phi_{2}\left(\tau_{l_{j-1}}\right) & \phi_{1}\left(\tau_{l_{j-1}}\right)\end{array}\right)$

second, the $j(j=1, \ldots, d)$ block of the subdiagonal, $\widetilde{\mathbf{0}}_{j}$, is a $\left(l_{j}-l_{j-1}\right) \times\left(l_{j+1}-l_{j}\right)$ matrix of zeros 
except for $\phi_{2}\left(\tau_{l_{j}}+1\right)$ in its $1 \times\left(l_{j+1}-l_{j}\right)$ entry, and third, the $j$ block of the superdiagonal $\overline{\mathbf{0}}_{j}$, is a $\left(l_{j+1}-l_{j}\right) \times\left(l_{j}-l_{j-1}\right)$ matrix of zeros except for -1 in its $\left(l_{j+1}-l_{j}\right) \times 1$ entry, and iv) there are zeros elsewhere.

\section{Abrupt Breaks}

Our general result has been presented in Section 2.3. In the current Section, we discuss still another example in order to both make our analysis clearer and to demonstrate its applicability. One important case is that of $r, 0 \leq r \leq k-1$, abrupt breaks at times $t-k_{1}, t-k_{2}, \ldots, t-k_{r}$, where $0=k_{0}<k_{1}<$ $k_{2}<\cdots<k_{r}<k_{r+1}=k, k_{r} \in \mathbb{Z}^{+}$, and $k_{r}$ is finite. That is, between $t-k=t-k_{r+1}$ and the present time $t=t-k_{0}$ the $\mathrm{AR}(2)$ process contains $r$ structural breaks and the switch from one set of parameters to another is abrupt. In particular

$$
y_{\tau}=\phi_{0, j}+\phi_{1, j} y_{\tau-1}+\phi_{2, j} y_{\tau-2}+\sigma_{j}^{2} e_{\tau, j}
$$

for $\tau=t-k_{j-1}, \ldots, t-k_{j}+1, j=1, \ldots, r+1$ and $e_{t, j} \sim$ i.i.d $(0,1) \forall t, j$. Within the class of $\operatorname{AR}(2)$ processes, this specification is quite general and allows for intercept and slope shifts as well as changes in the error variances (see also Pesaran et al., 2006). Each regime $j$ is characterized by a vector of autoregressive coefficients: $\phi_{0, j}, \phi_{j}^{\prime}=\left(\phi_{1, j}, \phi_{2, j}\right)$, and an error term variance, $0<\sigma_{j}^{2}<M_{j}<\infty \forall j$, $M_{j} \in \mathbb{R}^{+}$. We term this model abrupt breaks AR process of order $(2 ; r)(\operatorname{ABAR}(2 ; r))$.

For the $\operatorname{AR}(2)$ model with $r$ abrupt breaks, $\xi_{t, k}$ in eq. (6) can be written as the determinant of a partitioned (or a block) tridiagonal matrix

$$
\xi_{t, k}=\operatorname{det}\left(\boldsymbol{\Phi}_{t, k}\right)=\left|\begin{array}{lllll}
\boldsymbol{\Phi}_{t-k_{r}, k_{r+1}-k_{r}} & \overline{\mathbf{0}}_{r} & & & \\
\widetilde{\mathbf{0}}_{r} & \boldsymbol{\Phi}_{t-k_{r-1}, k_{r}-k_{r-1}} & \overline{\mathbf{0}}_{r-1} & & \\
& \ddots & \ddots & \ddots & \\
& & \widetilde{\mathbf{0}}_{2} & \boldsymbol{\Phi}_{t-k_{1}, k_{2}-k_{1}} & \overline{\mathbf{0}}_{1} \\
& & & \widetilde{\mathbf{0}}_{1} & \mathbf{\Phi}_{t, k_{1}}
\end{array}\right|,
$$

where first, the $j(j=1, \ldots, r+1)$ block of the main diagonal is $\mathbf{\Phi}_{t-k_{j-1}, k_{j}-k_{j-1}}$, 
that is a $\left(k_{j}-k_{j-1}\right) \times\left(k_{j}-k_{j-1}\right)$ banded Toeplitz matrix of bandwidth 3 :

$$
\boldsymbol{\Phi}_{t-k_{j-1}, k_{j}-k_{j-1}}=\left(\begin{array}{ccccc}
\phi_{1, j} & -1 & & & \\
\phi_{2, j} & \phi_{1, j} & -1 & & \\
& \ddots & \ddots & \ddots & \\
& & \phi_{2, j} & \phi_{1, j} & -1 \\
& & & \phi_{2, j} & \phi_{1, j}
\end{array}\right)
$$

with $\xi_{t-k_{j-1}, k_{j}-k_{j-1}}=\left|\mathbf{\Phi}_{t-k_{j-1}, k_{j}-k_{j-1}}\right|=\frac{1}{\lambda_{1, j}-\lambda_{2, j}}\left(\lambda_{1, j}^{k_{j}-k_{j-1}+1}-\lambda_{2, j}^{k_{j}-k_{j-1}+1}\right)$, and the second equality holds if and only if $\lambda_{1, j} \neq \lambda_{2, j}$ (where $1-\phi_{1, j} B-\phi_{2, j} B^{2}=\left(1-\lambda_{1, j} B\right)\left(1-\lambda_{2, j} B\right)$ ), second, the $j$ $(j=1, \ldots, r)$ block of the subdiagonal, $\widetilde{\mathbf{0}}_{j}$, is a $\left(k_{j}-k_{j-1}\right) \times\left(k_{j+1}-k_{j}\right)$ matrix of zeros except for $\phi_{2, j}$ in its $1 \times\left(k_{j+1}-k_{j}\right)$ entry, and third, the $j$ block of the superdiagonal $\overline{\mathbf{0}}_{j}$, is a $\left(k_{j+1}-k_{j}\right) \times\left(k_{j}-k_{j-1}\right)$ matrix of zeros except for -1 in its $\left(k_{j+1}-k_{j}\right) \times 1$ entry, and iv) there are zeros elsewhere.

Corollary 1 The general solution of the $A B A R(2 ; r)$ model in eq. (15) with free constants (initial condition values) $y_{t-k}, y_{t-k-1}$, is given by

$$
y_{t, k}^{g e n}=y_{t, k}^{h o m}+y_{t, k}^{p a r}
$$

where

$$
\begin{aligned}
y_{t, k}^{\text {hom }} & =\xi_{t, k} y_{t-k}+\phi_{2}(t-k+1) \xi_{t, k-1} y_{t-k-1} \\
y_{t, k}^{\text {par }} & =\sum_{j=1}^{r+1} \phi_{0, j} \sum_{i=k_{j-1}}^{k_{j}-1} \xi_{t, i}+\sum_{j=1}^{r+1} \sigma_{j}^{2} \sum_{i=k_{j-1}}^{k_{j}-1} \xi_{t, i} e_{t-i, j}
\end{aligned}
$$

and $\xi_{t, k}$ is given either in eq. (16) or in Proposition 7 (see Appendix B).

The proof of the above Corollary follows immediately from Theorem 1 and the definition of the $\operatorname{ABAR}(2 ; r)$ model in eq. (15).

\section{Prediction and Moment Structure}

We turn our attention to the fundamental properties of the various TV-AR(2) processes. Armed with a powerful technique for manipulating time varying models we may now provide a thorough description of the processes (1) by deriving, first, its multistep ahead predictor, the associated forecast error and the mean square error; second, the first two unconditional moments of this process, and third, its covariance 
structure.

\subsection{Multi Step Forecasts}

Taking the conditional expectation of eq. (7) with respect to the $\sigma$ field $F_{\tau_{k}}\left(\tau_{k}=t-k\right)$ yields the following Proposition.

Proposition 2 For the $T V$-AR(2) model the $k$-step-ahead optimal (in $L_{2}$-sense) linear predictor of $y_{t}$, $\mathbb{E}\left(y_{t} \mid F_{\tau_{k}}\right)$, is readily seen to be

$$
\mathbb{E}\left(y_{t} \mid F_{\tau_{k}}\right)=\sum_{i=0}^{k-1} \xi_{t, i} \phi_{0}(t-i)+\xi_{t, k} y_{t-k}+\phi_{2}(t-k+1) \xi_{t, k-1} y_{t-k-1}
$$

In addition, the forecast error for the above $k$-step-ahead predictor, $\mathbb{F} \mathbb{E}\left(y_{t} \mid F_{\tau_{k}}\right)=y_{t}-\mathbb{E}\left[y_{t} \mid F_{\tau_{k}}\right]$, is given by

$$
\mathbb{F} \mathbb{E}\left(y_{t} \mid F_{\tau_{k}}\right)=\Xi_{t, k}(B) \varepsilon_{t}=\sum_{i=0}^{k-1} \xi_{t, i} B^{i} \varepsilon_{t},
$$

and it is expressed in terms of $k$ error terms from time $t-k+1$ to time $t$; the coefficient of the error term at time $t-i, \xi_{t, i}$, is the determinant of an $i \times i$ matrix $\left(\Phi_{t, i}\right)$, each nonzero variable diagonal of which consists of the AR time varying coefficients $\phi_{m}(\cdot), m=1,2$ from time $t-i+m$ to $t$.

The mean square error is

$$
\operatorname{Var}\left[\mathbb{F} \mathbb{E}\left(y_{t} \mid F_{\tau_{k}}\right)\right]=\Xi_{t, k}^{(2)}(B) \sigma_{t}^{2}=\sum_{i=0}^{k-1} \xi_{t, i}^{2} B^{i} \sigma_{t}^{2}
$$

which is expressed in terms of $k$ variances from time $t-k+1$ to time $t$, with time varying coefficients (the squared $\xi s$ ).

The following Corollary presents results for the forecasts from PAR and CAR processes.

Corollary 2 For the $P A R(2 ; l)$ and the $C A R(2 ; l ; d)$ models (see eqs. (8) and (13) respectively) the nl-step-ahead optimal linear predictor is given by eq. 117) (with $k=n l$ ) in Proposition (2) where

$$
\begin{aligned}
\sum_{i=0}^{n l-1} \xi_{t, i} \phi_{0}(t-i) & =\sum_{s=0}^{l-1} \sum_{T=0}^{n-1} \xi_{t, T l+s} \phi_{0}(t-s) \quad(P A R \text { model }) \\
\sum_{i=0}^{n l-1} \xi_{t, i} \phi_{0}(t-i) & \left.=\sum_{j=1}^{d+1} \sum_{s_{j}=l_{j-1}+1}^{l_{j}} \sum_{T=0}^{n-1} \xi_{t, T l+s_{j}} \phi_{0}\left(t-s_{j}\right) \quad \text { (CAR model }\right),
\end{aligned}
$$

and $\xi_{t, T l+s}, \xi_{t, T l+s_{j}}$ are given in Proposition (6) and Corollary (3) respectively (see Appendix B).

Finally, for the $A B A R(2 ; r)$ model in eq. (15) the $k$-step-ahead optimal linear predictor is given by eq. 
17) where

$$
\sum_{i=0}^{k-1} \xi_{t, i} \phi_{0}(t-i)=\sum_{j=1}^{r+1} \phi_{0, j} \sum_{i=k_{j-1}}^{k j-1} \xi_{t, i}
$$

and $\xi_{t, i}$ is given either in eq. (16) or in Proposition (7) (see Appendix B).

Franses and Paap (2005) employ the vector season representation to compute forecasts and forecast error variances for a $\operatorname{PAR}(1 ; 4)$ process. In this way forecasts can be generated along the same lines with quadrivariate VAR(1) models. Franses (1996a) derives multi-step forecast error variances for low-order PAR models with $l=4$, using the VS representation. But, if $l$ is large even low order specifications will have large VAR representations and this is a handicap especially for forecasting. In contrast, our formulae using the univariate framework allow a fast computation of the multi-step-ahead predictors even if $l$ is large.

In what follows we give conditions for the first and second unconditional moments of model (1) to exist.

\subsection{Wold Representation}

First, we need an assumption.

Assumption A.1. $\sum_{i=0}^{k} \xi_{t, i} \phi_{0}(t-i)$ as $k \rightarrow \infty$ converges $\forall t$ and $\sum_{i=0}^{\infty} \sup _{t}\left(\xi_{t, i}^{2} \sigma_{t-i}^{2}\right)<M_{u}<\infty$ $\left(M_{u} \in \mathbb{R}^{+}\right)$.

Assumption A.1 is a sufficient condition for the model in eq. (11) to admit a second-order MA( $\infty)$ representation. A necessary but not sufficient condition for $\sum_{i=0}^{k} \xi_{t, i} \phi_{0}(t-i)$ to converge is $\lim _{k \rightarrow \infty}\left[\xi_{t, k} \phi_{0}(t-\right.$ $k)]=0 \forall t$. A sufficient condition for this limit to be zero is: $\lim _{k \rightarrow \infty} \xi_{t, k}=0$ and $\phi_{0}(t-k)$ is bounded.

Another immediate consequence of Theorem 1 is the following Proposition, where we state an expression for the first unconditional moment of $y_{t}$.

Proposition 3 Let Assumption A.1 hold. Then for the TV-AR(2) model we have:

$$
y_{t}=\lim _{k \rightarrow \infty} y_{t, k}^{g e n} \stackrel{L_{2}}{=} \lim _{k \rightarrow \infty} y_{t, k}^{\text {par }} \stackrel{L_{2}}{=} \Xi_{t, \infty}(B)\left[\phi_{0}(t)+\varepsilon_{t}\right]=\sum_{i=0}^{\infty} \xi_{t, i} B^{i}\left[\phi_{0}(t)+\varepsilon_{t}\right],
$$

is a unique solution of the $T V-A R(2)$ model in eq. (1). The above expression states that $\left\{y_{t, k}^{\text {par }}, t \in \mathbb{Z}\right\}$ (defined in eq. (7)) $L_{2}$ converges as $k \rightarrow \infty$ if and only if $\sum_{i=0}^{k} \xi_{t, i} \phi_{0}(t-i)$ converges and $\sum_{i=0}^{k} \xi_{t, i} \varepsilon_{t-i}$ converges a.s., and thus under assumption $A .1 \lim _{k \rightarrow \infty} y_{t, k}^{g e n} \stackrel{L_{2}}{=} \lim _{n \rightarrow \infty} y_{t, k}^{\text {par }}$ satisfies eq. (11).

In other words $\lim _{k \rightarrow \infty} y_{t, k}^{\text {gen }}$ is decomposed into a non random part and a zero mean random part. In 
particular,

$$
\mathbb{E}\left(y_{t}\right)=\lim _{k \rightarrow \infty} \mathbb{E}\left(y_{t} \mid F_{\tau_{k}}\right)=\Xi_{t, \infty}(B) \phi_{0}(t)=\sum_{i=0}^{\infty} \xi_{t, i} B^{i} \phi_{0}(t),
$$

is the non random part of $y_{t}$ and it is an infinite sum of the periodical drifts where the time varying coefficients are expressed as determinants of continuant matrices (the $\left.\xi_{s}\right)$, while $\lim _{k \rightarrow \infty} \mathbb{F} \mathbb{E}\left(y_{t} \mid F_{\tau_{k}}\right)=$ $\sum_{i=0}^{\infty} \xi_{t, i} \varepsilon_{t-i}$ is the zero mean random part. Therefore the $\xi_{t, i}$ as defined in eq. (6) are the Green functions associated with the second order time varying $A R$ polynomial: $\Phi_{t}(B)=1-\phi_{1}(t) B-\phi_{2}(t) B^{2}$.

\subsection{Second Moments}

In this subsection we state as a Proposition the result for the second moment structure.

Proposition 4 Let Assumption A.1 hold. Then the second unconditional moment of $y_{t}$ exists and it is given by

$$
\mathbb{E}\left(y_{t}^{2}\right)=\left[\mathbb{E}\left(y_{t}\right)\right]^{2}+\Xi_{t, \infty}^{(2)}(B) \sigma_{t}^{2}=\left[\mathbb{E}\left(y_{t}\right)\right]^{2}+\sum_{i=0}^{\infty} \xi_{t, i}^{2} B^{i} \sigma_{t}^{2} .
$$

That is, the time varying variance of $y_{t}$ is an infinite sum of the time varying variances of the errors with time varying coefficients (the squared values of the $\xi$ s).

In addition, the time varying autocovariance function $\gamma_{t, k}$ is given by

$$
\begin{aligned}
\gamma_{t, k}= & \operatorname{Cov}\left(y_{t}, y_{\tau_{k}}\right)=\sum_{i=0}^{\infty} \xi_{t, k+i} \xi_{\tau_{k}, i} \sigma_{\tau_{k}-i}^{2}=\xi_{t, k} \mathbb{V} \operatorname{ar}\left(y_{\tau_{k}}\right)+ \\
& \phi_{2}\left(\tau_{k}+1\right) \xi_{t, k-1} \mathbb{C o v}\left(y_{\tau_{k}}, y_{\tau_{k}-1}\right),
\end{aligned}
$$

where the second equality follows from the $M A(\infty)$ representation of $y_{t}$ in eq. (20) and the third one from eq. (7) in Theorem 1. For any fixed $t, \lim _{k \rightarrow \infty} \gamma_{t, k}=0$ when $\lim _{k \rightarrow \infty} \xi_{t, k}=0 \forall t$. Finally, recall that for the PAR and ABAR models the $\xi$ s are given either in eqs. (9) and (16) respectively, or in Propositions (6) and (7) respectively.

Although it may be difficult to compute the covariance structure of $\left\{y_{t}\right\}$ explicitly, for numerical work, one can always calculate it by computing the Green functions (that is, the continuant determinants $\xi$ s) with eqs. (5) and (6) and summing these with eq. (17).

\section{Conclusions}

We have provided the general solutions to low order TV-AR models in terms of their homogeneous and particular parts. Our first step was to find the fundamental set of solutions by computing the 
determinants of the matrix of coefficients associated with the infinite linear system that represents the difference equation.

The framework developed in Section 2, proved itself to be a general time varying theory, encompassing a number of seemingly unrelated models, discussed in Sections 3 and 4 . We have identified common properties (throughout the paper and in particular in Section 5), which are basic to each of the particular application.

We believe that time varying models should take center stage in the time series literature; this is why we have labored to develop a theory with rigorous foundations that can encompass a variety of dynamic systems, i.e., periodic and cyclical processes, and AR models which contain multiple structural breaks. Work that remains to be done by us and fellow researchers is on estimation and testing (for one application on this front see the paper by Karanasos et al., 2013) to demonstrate the usefulness of time varying models. In the long run, a sound mathematical theory has to be cointegrated with its applicability.

\section{REFERENCES}

Abdrabbo, N. A. and Priestley, M. B. (1967) On the prediction of non-stationary processes. Journal of the Royal Statistical Society, Series B 29, 570-85.

Adams, G. J. and Goodwin, G. C. (1995) Parameter estimation for periodic ARMA models. Journal of Time Series Analysis 16, 127-45.

Anderson, P. L., Meerschaert, M. M. and Zhang, K. (2013) Forecasting with prediction intervals for periodic autoregressive moving average models. Journal of Time Series Analysis 34, 187-93.

Anderson, P. L. and Vecchia, A. V. (1993) Asymptotic results for periodic autoregressive movingaverage processes. Journal of Time Series Analysis 14, 1-18.

Bentarzi, M. and Hallin, M. (1994) On the invertibility of periodic moving-average models. Journal of Time Series Analysis 15, 263-68.

Birchenhall, C. R., Bladen-Hovell, R. C., Chui, A. P. L., Osborn, D. R. and Smith, J. P. (1989) A seasonal model of consumption. The Economic Journal 99, 837-43.

Bollerslev, T. and Ghysels, E. (1996) Periodic autoregressive conditional heteroscedasticity. Journal of Business \& Economic Statistics 14, 139-51.

Cipra, T. and Tlustý, P. (1987) Estimation in multiple autoregressive-moving average models using periodicity. Journal of Time Series Analysis 8, 293-300.

del Barrio Castro, T. and Osborn, D. R. (2008) Testing for seasonal unit roots in periodic integrated autoregressive processes. Econometric Theory 24, 1093-129 
del Barrio Castro, T. and Osborn, D. R. (2012) Non-parametric testing for seasonally and periodically integrated processes. Journal of Time Series Analysis 33, 424-37.

Franses, P. H. (1994) A multivariate approach to modeling univariate seasonal time series. Journal of Econometrics 63, 133-51.

Franses, P. H. (1996a) Multi-step forecast error variances for periodically integrated time series. Journal of Forecasting 15, 83-95.

Franses, P. H. (1996b) Periodicity and Stochastic Trends in Economic Time Series. Oxford University Press.

Franses, P. H. and Paap, R. (2004) Periodic Time Series models. Oxford. Oxford University Press.

Franses, P. H. and Paap, R. (2005) Forecasting with periodic autoregressive time-series models, in A Companion to Economic Forecasting, Eds. Clements, M. P. and Hendry, D. F. Oxford. Wiley-Blackwell, pp. $432-52$.

Ghysels, E. and Osborn, D. R. (2001) The Econometric Analysis of Seasonal Time Series. Cambridge University Press.

Gladyshev, E. G. (1961) On periodically correlated random sequences. Soviet Mathematics 2, 385-88.

Granger, C. W. J. (2007) Forecasting - looking back and forward: Paper to celebrate the 50th anniversary of the econometrics institute at the Erasmus University, Rotterdam. Journal of Econometrics 138, 3-13.

Granger, C. W. J. (2008) Non-linear models: Where do we go next - time varying parameter models? Studies in Nonlinear Dynamics and Econometrics 12, 1-9.

Hurd, H. L. and Miamee, A. (2007) Periodically Correlated Random Sequences: Spectral Theory and Practice. Hoboken, New Jersey. Wiley-Blackwell.

Karanasos, M. (2001) Prediction in ARMA models with GARCH in mean effects. Journal of Time Series Analysis 22, 555-76.

Karanasos, M., Paraskevopoulos, A. G. and Dafnos, S. (2013) A univariate time varying analysis of periodic ARMA processes. Unpublished Paper.

Karanasos, M., Paraskevopoulos, A. G., Menla Ali, F., Karoglou, M. and Yfanti, S. (2013) Modelling returns and volatilities during financial crises: a time varying coefficient approach. Unpublished Paper.

Lund, R. and Basawa, I. V. (2000) Recursive prediction and likelihood evaluation for periodic ARMA models. Journal of Time Series Analysis 21, 75-93.

Lund, R., Shao, Q. and Basawa, I. (2006) Parsimonious periodic time series modeling. Australian 86 New Zealand Journal of Statistics 48, 33-47.

McLeod, A. I. (1994) Diagnostic checking of periodic autoregression models with application. Journal 
of Time Series Analysis 15, 221-33.

Osborn, D. R. (1988) Seasonality and habit persistence in a life cycle model of consumption. Journal of Applied Econometrics 3, 255-66.

Osborn, D. R. (1991) The implications of periodically varying coefficients for seasonal time-series processes. Journal of Econometrics 48, 373-84.

Osborn, D. R. and Smith, J. P. (1989) The performance of periodic autoregressive models in forecasting seasonal U.K. consumption. Journal of Business 6 Economic Statistics 7, 117-27.

Pagano, M. (1978) On periodic and multiple autoregressions. The Annals of Statistics 6, 1310-317.

Paraskevopoulos, A. G. (2012) The Infinite Gauss-Jordan elimination on row-finite $\omega \times \omega$ matrices. arXiv: 1201.2950 .

Paraskevopoulos, A. G. and Karanasos, M. (2013) Closed form solutions for linear difference equations with time dependent coefficients. Unpublished paper.

Paraskevopoulos, A. G., Karanasos, M. and Dafnos, S. (2013) A unified theory for time varying models: foundations with applications in the presence of breaks and heteroskedasticity (and some results on companion and Hessenberg matrices). Unpublished Paper.

Pesaran, M. H., Pettenuzzo, D. and Timmermann, A. (2006) Forecasting time series subject to multiple structural breaks. Review of Economic Studies 73, 1057-084.

Rao, S. T. (1970) The fitting of non-stationary time-series models with time-dependent parameters. Journal of the Royal Statistical Society, Series B 32, 312-22.

Shao, Q. (2008) Robust estimation for periodic autoregressive time series. Journal of Time Series Analysis 29, 251-63.

Sydsaeter, K., Hammond, P. J., Seierstad, A. and Strom, A. (2008) Further Mathematics for Economic Analysis. New York. Prentice Hall, 2nd edition.

Taylor, A. M. R. (2002) Regression-based unit root tests with recursive mean adjustment for seasonal and nonseasonal time series. Journal of Business E Economic Statistics 20, 269-81.

Taylor, A. M. R. (2003) On the asymptotic properties of some seasonal unit root tests. Econometric Theory 19, 311-21.

Taylor, A. M. R. (2005) Variance ratio tests of the seasonal unit root hypothesis. Journal of Econometrics 124, 33-54.

Tesfaye Y. G., Anderson, P. L. and Meerschaert, M. M. (2011) Asymptotic results for Fourier-PARMA time series. Journal of Time Series Analysis 32, 157-74.

Tiao, G. C. and Grupe, M. R. (1980) Hidden periodic autoregressive-moving average models in time series data. Biometrika 67, 365-73. 
Tiao, G. C. and Guttman, I. (1980) Forecasting contemporal aggregates of multiple time series. Journal of Econometrics 12, 219-30.

Whittle, P. (1965) Recursive relations for predictors of non-stationary processes. Journal of the Royal Statistical Society, Series B 27, 523-32.

\section{A APPENDIX}

In this appendix we prove Theorem 1. Before proceeding with the main body of the proof, we present two essential tools for carrying it out.

The Infinite Gaussian Elimination. Following Paraskevopoulos (2012), we apply the infinite Gaussian elimination algorithm implemented under a rightmost pivot strategy to the coefficient matrix $\Phi$ of (3). The process is briefly described below.

Call $\mathbf{h}^{(1)}=\mathbf{H}^{(1)}=\left(-\phi_{2}\left(\tau_{k}+1\right),-\phi_{1}\left(\tau_{k}+1\right), 1,0, \ldots\right)$ the opposite-sign first row of $\boldsymbol{\Phi}$. Insert the second row of $\boldsymbol{\Phi}$ below $\mathbf{H}^{(1)}$ to build the matrix $\mathbf{B}^{(2)}$ :

$$
\mathbf{B}^{(2)}=\left(\begin{array}{ccccc}
-\phi_{2}\left(\tau_{k}+1\right) & -\phi_{1}\left(\tau_{k}+1\right) & 1 & 0 & \ldots \\
0 & \phi_{2}\left(\tau_{k}+2\right) & \phi_{1}\left(\tau_{k}+2\right) & -1 & \ldots
\end{array}\right)
$$

Use as pivot the rightmost one of $\mathbf{H}^{(1)}$ to clear the element $\phi_{1}\left(\tau_{k}+2\right)$ in the second row of $\mathbf{B}^{(2)}$. After normalization it yields the matrix:

$$
\mathbf{H}^{(2)}=\left(\begin{array}{ccccc}
-\phi_{2}\left(\tau_{k}+1\right) & -\phi_{1}\left(\tau_{k}+1\right) & 1 & 0 & \ldots \\
-\phi_{2}\left(\tau_{k}+1\right) \phi_{1}\left(\tau_{k}+2\right) & -\phi_{2}\left(\tau_{k}+2\right)-\phi_{1}\left(\tau_{k}+1\right) \phi_{1}\left(\tau_{k}+2\right) & 0 & 1 & \ldots
\end{array}\right) .
$$

Insert the third row of $\boldsymbol{\Phi}$ below $\mathbf{H}^{(2)}$ to build the matrix $\mathbf{B}^{(3)}$ :

$$
\left(\begin{array}{cccccc}
-\phi_{2}\left(\tau_{k}+1\right) & -\phi_{1}\left(\tau_{k}+1\right) & 1 & 0 & 0 & \ldots \\
-\phi_{2}\left(\tau_{k}+1\right) \phi_{1}\left(\tau_{k}+2\right) & -\phi_{2}\left(\tau_{k}+2\right)-\phi_{1}\left(\tau_{k}+1\right) \phi_{1}\left(\tau_{k}+2\right) & 0 & 1 & 0 & \ldots \\
0 & 0 & \phi_{2}\left(\tau_{k}+3\right) & \phi_{1}\left(\tau_{k}+3\right) & -1 & \ldots
\end{array}\right)
$$

Use the first two rows of $\mathbf{B}^{(3)}$ as pivot rows and their rightmost 1s as pivot elements to clear the entries $\phi_{2}\left(\tau_{k}+3\right)$ and $\phi_{1}\left(\tau_{k}+3\right)$ of $\mathbf{B}^{(3)}$, producing the matrix $\mathbf{H}^{(3)}$ :

$$
\mathbf{H}^{(3)}=\left(\begin{array}{ccccccc}
h_{11} & h_{12} & 1 & 0 & 0 & 0 & \ldots \\
h_{21} & h_{22} & 0 & 1 & 0 & 0 & \ldots \\
h_{31} & h_{32} & 0 & 0 & 1 & 0 & \ldots
\end{array}\right)
$$


where the entries of the first column of $\mathbf{H}^{(3)}$ are given by

$$
\begin{aligned}
& h_{11}=-\phi_{2}\left(\tau_{k}+1\right), h_{21}=-\phi_{2}\left(\tau_{k}+1\right) \phi_{1}\left(\tau_{k}+2\right), \\
& h_{31}=-\phi_{2}\left(\tau_{k}+1\right) \phi_{1}\left(\tau_{k}+2\right) \phi_{1}\left(\tau_{k}+3\right)-\phi_{2}\left(\tau_{k}+1\right) \phi_{2}\left(\tau_{k}+3\right), \ldots
\end{aligned}
$$

and the entries of the second column are given by

$$
\begin{aligned}
& h_{12}=-\phi_{1}\left(\tau_{k}+1\right), h_{22}=-\phi_{2}\left(\tau_{k}+2\right)-\phi_{1}\left(\tau_{k}+1\right) \phi_{1}\left(\tau_{k}+2\right), \\
& h_{32}=-\phi_{1}\left(\tau_{k}+1\right) \phi_{1}\left(\tau_{k}+2\right) \phi_{1}\left(\tau_{k}+3\right)-\phi_{2}\left(\tau_{k}+2\right) \phi_{1}\left(\tau_{k}+3\right)-\phi_{2}\left(\tau_{k}+3\right) \phi_{1}\left(\tau_{k}+1\right)
\end{aligned}
$$

This process continues ad infinitum, generating an infinite chain of submatrices

$$
\mathbf{H}^{(1)} \sqsubset \mathbf{H}^{(2)} \sqsubset \mathbf{H}^{(3)} \sqsubset \ldots \sqsubset \mathbf{H}
$$

whose limit row-finite matrix $\mathbf{H}$ is the Hermite Form (HF) of $\mathbf{\Phi}$. The $i$ th row of $\mathbf{H}$ is defined to be the last row of $\mathbf{H}^{(i)}$.

Two Fundamental Solutions. The opposite-sign two first columns of $\mathbf{H}$ augmented at the top by $(1,0)$ and $(0,1)$, respectively, that is

$$
\begin{aligned}
& \boldsymbol{\xi}_{\tau_{k}}^{(2)}=\left(1, \quad 0, \quad \phi_{2}\left(\tau_{k}+1\right), \quad \phi_{2}\left(\tau_{k}+1\right) \phi_{1}\left(\tau_{k}+2\right),\right. \\
& \left.\phi_{2}\left(\tau_{k}+1\right) \phi_{1}\left(\tau_{k}+2\right) \phi_{1}\left(\tau_{k}+3\right)+\phi_{2}\left(\tau_{k}+1\right) \phi_{2}\left(\tau_{k}+3\right), \ldots\right)^{\prime} \text {, }
\end{aligned}
$$

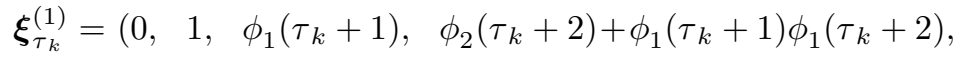

$$
\begin{aligned}
& \left.\phi_{1}\left(\tau_{k}+1\right) \phi_{1}\left(\tau_{k}+2\right) \phi_{1}\left(\tau_{k}+3\right)+\phi_{2}\left(\tau_{k}+2\right) \phi_{1}\left(\tau_{k}+3\right)+\phi_{2}\left(\tau_{k}+3\right) \phi_{1}\left(\tau_{k}+1\right), \ldots\right)^{\prime}
\end{aligned}
$$

are the two linearly independent solution sequences of the space of homogeneous solutions of eq. (2). The linear independence of $\boldsymbol{\xi}_{\tau_{k}}^{(1)}, \boldsymbol{\xi}_{\tau_{k}}^{(2)}$ follows from the fact that they possess the Casoratian:

$$
\operatorname{det}\left(\begin{array}{ll}
1 & 0 \\
0 & 1
\end{array}\right) \neq 0
$$


We observe that the terms of the sequences $\boldsymbol{\xi}_{\tau_{k}}^{(1)}, \boldsymbol{\xi}_{\tau_{k}}^{(2)}$ are expansions of the following determinants

$$
\begin{aligned}
& \boldsymbol{\xi}_{\tau_{k}}^{(2)}=\left\{\begin{array}{l}
1 \\
0 \\
\operatorname{det}\left(\begin{array}{cc}
\phi_{2}\left(\tau_{k}+1\right) & -1 \\
0 & \phi_{1}\left(\tau_{k}+2\right)
\end{array}\right) \\
\operatorname{det}\left(\begin{array}{ccc}
\phi_{2}\left(\tau_{k}+1\right) & -1 & \\
0 & \phi_{1}\left(\tau_{k}+2\right) & -1 \\
0 & \phi_{2}\left(\tau_{k}+3\right) & \phi_{1}\left(\tau_{k}+3\right)
\end{array}\right), \\
\cdot \\
\cdot
\end{array}\right. \\
& \boldsymbol{\xi}_{\tau_{k}}^{(1)}=\left\{\begin{array}{l}
0 \\
1 \\
\phi_{1}\left(\tau_{k}+1\right) \\
\operatorname{det}\left(\begin{array}{cc}
\phi_{1}\left(\tau_{k}+1\right) & -1 \\
\phi_{2}\left(\tau_{k}+2\right) & \phi_{1}\left(\tau_{k}+2\right)
\end{array}\right) \\
\operatorname{det}\left(\begin{array}{ccc}
\phi_{1}\left(\tau_{k}+1\right) & -1 \\
\phi_{2}\left(\tau_{k}+2\right) & \phi_{1}\left(\tau_{k}+2\right) & -1 \\
0 & \phi_{2}\left(\tau_{k}+3\right) & \phi_{1}\left(\tau_{k}+3\right)
\end{array}\right) \\
\cdot \cdot \begin{array}{cc} 
\\
\cdot \\
\cdot
\end{array}
\end{array}\right.
\end{aligned}
$$

The first few terms of the homogeneous solution sequences, as shown above, suggest that the general terms of $\boldsymbol{\xi}_{\tau_{k}}^{(1)}, \boldsymbol{\xi}_{\tau_{k}}^{(2)}$ are

$$
\xi_{t, k}^{(m)}=\operatorname{det}\left(\boldsymbol{\Phi}_{t, k}^{(m)}\right), \quad m=1,2,
$$

where $\boldsymbol{\Phi}_{t, k}^{(1)}=\boldsymbol{\Phi}_{t, k}$ and $\xi_{t, k}^{(1)}=\xi_{t, k}$ (we drop the superscript 1 for notational convenience), as introduced 
in eqs. (5) and (6), and

$$
\boldsymbol{\Phi}_{t, k}^{(2)}=\left(\begin{array}{cccccc}
\phi_{2}\left(\tau_{k}+1\right) & -1 & & & & \\
& \phi_{1}\left(\tau_{k}+2\right) & -1 & & & \\
& \phi_{2}\left(\tau_{k}+3\right) & \phi_{1}\left(\tau_{k}+3\right) & -1 & & \\
& & \ddots & \ddots & \ddots & \\
& & & \phi_{2}(t-1) & \phi_{1}(t-1) & -1 \\
& & & & \phi_{2}(t) & \phi_{1}(t)
\end{array}\right) .
$$

In the following Proposition we use mathematical induction to verify the above generalization formally.

Proposition 5 The general terms of the fundamental solution sequences $\boldsymbol{\xi}_{\tau_{k}}^{(m)}, m=1,2$, are given by eq. (A.3), that is

$$
\xi_{t, k}^{(2)}=\operatorname{det}\left(\begin{array}{cccccc}
\phi_{2}\left(\tau_{k}+1\right) & -1 & & & & \\
& \phi_{1}\left(\tau_{k}+2\right) & -1 & & & \\
& \phi_{2}\left(\tau_{k}+3\right) & \phi_{1}\left(\tau_{k}+3\right) & -1 & & \\
& & \ddots & \ddots & \ddots & \\
& & & \phi_{2}(t-1) & \phi_{1}(t-1) & -1 \\
& & & & \phi_{2}(t) & \phi_{1}(t)
\end{array}\right)
$$

and

$$
\xi_{t, k}=\operatorname{det}\left(\begin{array}{cccccc}
\phi_{1}\left(\tau_{k}+1\right) & -1 & & & & \\
\phi_{2}\left(\tau_{k}+2\right) & \phi_{1}\left(\tau_{k}+2\right) & -1 & & & \\
& \phi_{2}\left(\tau_{k}+3\right) & \phi_{1}\left(\tau_{k}+3\right) & -1 & & \\
& & \ddots & \ddots & \ddots & \\
& & & \phi_{2}(t-1) & \phi_{1}(t-1) & -1 \\
& & & & \phi_{2}(t) & \phi_{1}(t)
\end{array}\right) \text {. }
$$

Proof. If $t=\tau_{k}+1$ and $t=\tau_{k}+2$ then $\xi_{\tau_{k}+1,1}$ and $\xi_{\tau_{k}+2,2}$ is the third term and fourth term of the sequences as directly verified by eq. (A.2). We assume that $\xi_{t-2, k-2}$ and $\xi_{t-1, k-1}$ are terms of $\boldsymbol{\xi}_{\tau_{k}}^{(1)}$. We show that $\xi_{t, k}$ is also a term of $\boldsymbol{\xi}_{\tau_{k}}^{(1)}$. Expanding $\xi_{t, k}$ along the last row and taking into account that $\boldsymbol{\Phi}_{t, k}$ 
is a $k \times k$ matrix, we have:

$$
\xi_{t, k}=(-1)^{2 k} \phi_{1}(t) \operatorname{det}\left(\begin{array}{cccccc}
\phi_{1}\left(\tau_{k}+1\right) & -1 & & & & \\
\phi_{2}\left(\tau_{k}+2\right) & \phi_{1}\left(\tau_{k}+2\right) & -1 & & & \\
& \phi_{2}\left(\tau_{k}+3\right) & \phi_{1}\left(\tau_{k}+3\right) & -1 & & \\
& & \ddots & \ddots & \ddots & \\
& & & \phi_{2}(t-2) & \phi_{1}(t-2) & -1 \\
& & & & \phi_{2}(t-1) & \phi_{1}(t-1)
\end{array}\right)+
$$

Using the induction hypothesis, the above result can be written as

$$
\xi_{t, k}=\phi_{1}(t) \xi_{t-1, k-1}+\phi_{2}(t) \xi_{t-2, k-2}
$$

which shows that $\xi_{t, k}$ is a homogeneous solution of (2). Thus $\xi_{t, k}$ in (A.5) is a term of the solution sequence and the induction is complete. By analogy, we can show (A.4) and the proof is complete.

The fundamental solution $\xi_{t, k}$ (respectively $\xi_{t, k}^{(2)}$ ) can be obtained by augmenting the core solution matrix $\mathbf{C}_{t, k}$ (see eq. (4) in the main body of the paper) on the left by a $k \times 1$ column consisting of the first $k$ entries of the second column (respectively of the first column) of $\mathbf{P}$ or $\boldsymbol{\Phi}$.

Proof. (of Theorem 1) As a direct consequence of Proposition 1, the general homogeneous solution of eq. (2) is the linear combination of the fundamental solutions as given below:

$$
y_{t, k}^{h o m}=\xi_{t, k} y_{\tau_{k}}+\xi_{t, k}^{(2)} y_{\tau_{k}-1}
$$

By expanding $\xi_{t, k}^{(2)}$ along the first column we obtain

$$
\xi_{t, k}^{(2)}=\phi_{2}\left(\tau_{k}+1\right) \xi_{t, k-1}
$$


and therefore (A.6) takes the form

$$
y_{t, k}^{h o m}=\xi_{t, k} y_{\tau_{k}}+\phi_{2}\left(\tau_{k}+1\right) \xi_{t, k-1} y_{\tau_{k}-1}
$$

which coincides with the general homogeneous solution employed in eq. (7).

Next we show that $y_{t, k}^{p a r}$, employed in eq. (77), is a particular solution of eq. (2). Using the same arguments as in the proof of Proposition 5 we can show that

$$
y_{t, k}^{p a r}=\operatorname{det}\left(\begin{array}{cccccc}
\phi_{0}\left(\tau_{k}+1\right)+\epsilon_{\tau_{k}+1} & -1 & & & & \\
\phi_{0}\left(\tau_{k}+2\right)+\epsilon_{\tau_{k}+2} & \phi_{1}\left(\tau_{k}+2\right) & -1 & & & \\
\phi_{0}\left(\tau_{k}+3\right)+\epsilon_{\tau_{k}+3} & \phi_{2}\left(\tau_{k}+3\right) & \phi_{1}\left(\tau_{k}+3\right) & -1 & & \\
\vdots & & \ddots & \ddots & \ddots & \\
\phi_{0}(t-1)+\epsilon_{t-1} & & & \phi_{2}(t-1) & \phi_{1}(t-1) & -1 \\
\phi_{0}(t)+\epsilon_{t} & & & & \phi_{2}(t) & \phi_{1}(t)
\end{array}\right) \text {, }
$$

is the solution of the initial value problem determined by eq. (2) subject to the initial values $y_{-1}=y_{0}=0$. This is the determinant of the core solution matrix $\mathbf{C}_{t, k}$ augmented on the left by a $k \times 1$ column consisting of the opposite sign first $k$ entries of the right-hand side sequence of eq. (2).

Now expanding the determinant in eq. (A.7) along the first column we obtain $y_{t, k}^{p a r}$ in terms of $\xi_{t, i}$ and $\phi_{0}(t-i), \epsilon_{t-i}$ for $i=0,1, \ldots, k-1$, as used in eq. (7). Therefore the general solution in eq. (7), as the sum of the general homogeneous solution plus a particular solution, has been established. This completes the proof of Theorem 1.

\section{B APPENDIX}

In this Appendix we will make use of the block Toeplitz matrix in eq. (10) to obtain an explicit formula of $\xi_{t, n l}$ in which we decompose it into tridiagonal determinants, $\xi_{t, l}$. To prepare the reader, before we present the main result we consider the case where $n=2$, that is we go from time $t$ back to time $t-2 l$. The tridiagonal determinant $\xi_{t, 2 l}$ can be written as the sum of two terms

$$
\begin{aligned}
\xi_{t, 2 l} & =\left|\begin{array}{cc}
\boldsymbol{\Phi}_{t, l} & \overline{\mathbf{0}} \\
\widetilde{\mathbf{0}}_{t} & \boldsymbol{\Phi}_{t, l}
\end{array}\right|= \\
& =\xi_{t, l}^{2}+\phi_{2}\left(\tau_{l}+1\right) \xi_{t, l-1} \xi_{t-1, l-1},
\end{aligned}
$$


where each term is the product of two continuant (or tridiagonal) determinants.

Next let $i_{j} \in\{0,1\}, j=1, \ldots, n-1$, and define

$$
\varphi_{2, j}=\left\{\begin{array}{ccc}
1 & \text { if } & i_{j}=0 \\
\phi_{2}\left(\tau_{j l}+1\right) & \text { if } & i_{j}=1
\end{array}\right.
$$

Proposition 6 For the PAR(2;l) process in eq. (8), $\xi_{t, n l}$ is the determinant of $\boldsymbol{\Phi}_{t, n l}$ in eq. (9), and therefore can be written as

$$
\xi_{t, n l}=\sum_{i_{1}=0}^{1} \cdots \sum_{i_{n-1}=0}^{1}\left\{\xi_{t, l-i_{1}}\left(\prod_{T=2}^{n-1} \varphi_{2, T-1} \xi_{t-i_{T-1}, l-i_{T}-i_{T-1}}\right) \varphi_{2, n-1} \xi_{t-i_{n-1}, l-i_{n-1}}\right\}
$$

where $\sum \cdots \sum$ stands for a multiple but finite summation, and recall that $\xi_{t, l}=\left|\boldsymbol{\Phi}_{t, l}\right|$ and $\boldsymbol{\Phi}_{t, l}$ is given by eq. (111).

In the above Proposition $\xi_{t, n l}$ is expressed as the sum of $\sum_{j=0}^{n-1}\left(\begin{array}{c}n-1 \\ j\end{array}\right)=2^{n-1}$ terms, each of which is the product of $n$ terms. In other words, it is decomposed into determinants of $(l-m) \times(l-m)$ continuant matrices, $m=0,1,2: \Phi_{t-i_{T-1}, l-i_{T}-i_{T-1}}$.

When $n=3$, eq. (B.3) reduces to:

$$
\begin{aligned}
\xi_{t, n l}= & \xi_{t, l}^{3}+\phi_{2}\left(\tau_{l}+1\right) \xi_{t, l-1} \xi_{t-1, l-1} \xi_{t, l} \\
& +\xi_{t, l} \phi_{2}\left(\tau_{l}+1\right) \xi_{t, l-1} \xi_{t-1, l-1} \\
& +\phi_{2}^{2}\left(\tau_{l}+1\right) \xi_{t, l-1} \xi_{t-1, l-2} \xi_{t-1, l-1} \\
= & \xi_{t, l}^{3}+2 \phi_{2}\left(\tau_{l}+1\right) \xi_{t, l-1} \xi_{t-1, l-1} \xi_{t, l}+\phi_{2}^{2}\left(\tau_{l}+1\right) \xi_{t, l-1} \xi_{t-1, l-2} \xi_{t-1, l-1},
\end{aligned}
$$

that is, $\xi_{t, n l}$ is equal to the sum of four $\left(p^{n-1}=2^{2} ; i_{1}=i_{2}=0, i_{1}=i_{2}=1, i_{1}=0\right.$ and $i_{2}=1$, and $i_{1}=1$ and $\left.i_{2}=0\right)$ terms, each of which is the product of three $(n=3) \xi^{\prime}$ 's (continuant determinants).

Next we will prove Proposition 6 by mathematical induction. For $n=2$ the result has been proved in eq. (B.1). If we assume that eq. (B.3) holds for $n$ then it will be sufficient to prove that it holds for $n+1$ as well.

Proof. (Proposition 6) Assume that

$$
\xi_{t, n l}=\left|\Phi_{t, n l}\right|=\sum_{i_{1}=0}^{1} \cdots \sum_{i_{n-1}=0}^{1}\left\{\xi_{t, l-i_{1}}\left(\prod_{l=2}^{n-1} \varphi_{2, T-1} \xi_{t-i_{T-1}, l-i_{T}-i_{T-1}}\right) \varphi_{2, n-1} \xi_{t-i_{n-1}, l-i_{n-1}}\right\}
$$


Similarly to eq. (B.1) we can express $\xi_{t,(n+1) l}$ as the determinant of a $2 \times 2$ block matrix:

$$
\begin{aligned}
\xi_{t,(n+1) l} & =\left|\begin{array}{cl}
\boldsymbol{\Phi}_{t, l} & \overline{\mathbf{0}} \\
\widetilde{\mathbf{0}}_{t} & \boldsymbol{\Phi}_{t, n l}
\end{array}\right|=\left|\boldsymbol{\Phi}_{t, n l}\right|\left|\boldsymbol{\Phi}_{t, l}\right|+\phi_{2}(t-n l+1)\left|\boldsymbol{\Phi}_{t, n l-1}\right|\left|\mathbf{\Phi}_{t-1, l-1}\right| \\
& =\xi_{t, n l} \xi_{t, l}+\phi_{2}(t-n l+1) \xi_{t, n l-1} \xi_{t-1, l-1},
\end{aligned}
$$

where $\widetilde{\mathbf{0}}_{t}$, is an $n l \times l$ matrix of zeros except for $\phi_{2}(t-n l+1)$ in its $1 \times l$ entry and the second equality follows from eq. (B.1). Combining eqs. (B.4) and (B.5) yields

$$
\begin{aligned}
\xi_{t,(n+1) l}=\sum_{i_{1}=0}^{1} \cdots \sum_{i_{n-1}=0}^{1}\left\{\xi_{t, l-i_{1}}\left(\prod_{T=2}^{n-1} \varphi_{2, T-1} \xi_{t-i_{T-1}, l-i_{T}-i_{T-1}}\right) \varphi_{2, n-1} \xi_{t-i_{n-1}, l-i_{n-1}}\right\} \xi_{t, l}+ \\
\sum_{i_{1}=0}^{1} \cdots \sum_{i_{n-1}=0}^{1}\left\{\xi_{t, l-i_{1}}\left(\prod_{T=2}^{n-1} \varphi_{2, T-1} \xi_{t-i_{T-1}, l-i_{T}-i_{T-1}}\right) \varphi_{2, n-1} \xi_{t-i_{n-1}, l-1-i_{n-1}}\right\} \phi_{2}(t-n l+1) \xi_{t-1, l-1} \\
=\sum_{i_{1}=0}^{1} \cdots \sum_{i_{n}=0}^{1}\left\{\xi_{t, l-i_{1}}\left(\prod_{T=2}^{n} \varphi_{2, T-1} \xi_{t-i_{T-1}, l-i_{T}-i_{T-1}}\right) \varphi_{2, n} \xi_{t-i_{n}, l-i_{n}}\right\}
\end{aligned}
$$

which completes the proof.

Corollary 3 For the $C A R(2 ; l ; d)$ process, in eq. 113), with $0 \leq d \leq l-1, \xi_{t, l}=\left|\Phi_{t, l}\right|$ (see eq. 144)) can be written as

$$
\xi_{t, l}=\sum_{i_{1}=0}^{1} \cdots \sum_{i_{d}=0}^{1}\left\{\xi_{t, l_{1}-i_{1}}\left(\prod_{j=2}^{d} \varphi_{2, j-1} \xi_{t-l_{j-1}-i_{j-1}, l_{j}-l_{j-1}-i_{j}-i_{j-1}}\right) \varphi_{2, d} \xi_{t-l_{d}-i_{d}, l-l_{d}-i_{d}}\right\}
$$

where $\varphi_{2, j}$ is defined similarly to the one in eq. (B.2), i.e., $\varphi_{2, j}=\phi_{2}\left(t-\left(l_{j}-l_{j-1}\right)+1\right)$ if $i_{j}=1$ (the proof is along the lines of that of Proposition (6) above).

Proposition 7 For the $A B A R(2 ; r)$ process in eq. (15) with $r, 0 \leq r \leq k-1$, abrupt breaks at times $t-k_{1}, t-k_{2}, \ldots, t-k_{r}, \xi_{t, k}$ in eq. (16) can be written as

$$
\xi_{t, k}=\sum_{i_{1}=0}^{1} \cdots \sum_{i_{r}=0}^{1}\left\{\xi_{t, k_{1}-i_{1}}\left(\prod_{j=2}^{r} \varphi_{2, j-1} \xi_{t-k_{j-1}-i_{j-1}, k_{j}-k_{j-1}-i_{j}-i_{j-1}}\right) \varphi_{2, r} \xi_{t-k_{r}-i_{r}, k-k_{r}-i_{r}}\right\} .
$$

where $\varphi_{2, j}$ is defined similarly to the one in eq. (B.2) (the proof is similar to that of Proposition (6) above).

\section{APPENDIX}

Vector Seasons Representation 
For the benefit of the reader this Appendix reviews some results on PARMA models. Recall that the drift and the autoregressive coefficients are periodically varying: $\phi_{m}(t)=\phi_{m}\left(\tau_{n}\right), m=0,1,2$, $\tau_{n}=\tau-n l$. Recall also that $t_{s}$ denotes time at the $s$ th season: $t_{s}=T l+s, s=1, \ldots, l$, and that we can write $\phi_{m}(T l+s)=\phi_{m, s}$ (see eq. (8) ).

We assume without loss of generality that time $t$ is at the $l$ th season (e.g., $t=t_{l}=(T+1) l$ ). Thus our $\boldsymbol{\Phi}_{\mathrm{t}, l}$ matrix in eq. (11) becomes:

$$
\boldsymbol{\Phi}_{t, l}=\boldsymbol{\Phi}(l)=\left(\begin{array}{ccccc}
\phi_{1,1} & -1 & & & \\
\phi_{2,2} & \phi_{1,2} & -1 & & \\
& \ddots & \ddots & \ddots & \\
& & \phi_{2, l-1} & \phi_{1, l-1} & -1 \\
& & & \phi_{2, l} & \phi_{1, l}
\end{array}\right) .
$$

A convenient representation of the $\operatorname{PAR}(2 ; l)$ model (8) is the $\operatorname{VAR}(1)$ representation- hereafter we will refer to it as the vector of seasons (VS) representation (see, for example, Tiao and Guttman, 1980; Osborn, 1991; Franses, 1994, 1996a,b; del Barrio Castro and Osborn, 2008).

The corresponding VS representation of the $\operatorname{PAR}(2 ; l)$ model (ignoring the drifts) is given by

$$
\mathbf{\Phi}_{0} \mathbf{y}_{T}=\boldsymbol{\Phi}_{1} \mathbf{y}_{T-1}+\varepsilon_{T}
$$

with $\mathbf{y}_{T}=\left(y_{1 T}, \ldots, y_{l T}\right)^{\prime}, \boldsymbol{\varepsilon}_{T}=\left(\varepsilon_{1 T}, \ldots, \varepsilon_{l T}\right)^{\prime}$, where the first subscript refers to the season $(s)$ and the second one to the 'period' $(T)$. Moreover, $\boldsymbol{\Phi}_{0}$ is an $l \times l$ parameter matrix whose $(i, j)$ entry is:

$$
\left\{\begin{array}{lll}
1 & \text { if } & i=j, \\
0 & \text { if } & j>i \\
-\phi_{i-j, i} & \text { if } & j<i
\end{array}\right.
$$

and $\boldsymbol{\Phi}_{1}$ is an $l \times l$ parameter matrix with $(i, j)$ elements $\phi_{i+l-j, i}$, (see, for example, Lund and Basawa, 2000, and Franses and Paap, 2005).

As pointed out by Franses (1994), the idea of stacking was introduced by Gladyshev (1961) and is also considered in e.g., Pagano (1978). Tiao and Guttman (1980), Osborn (1991) and Franses (1994) used it in the AR setting. The dynamic system in eq. (C.1) can be written in a compact form

$$
\mathbf{\Phi}(B) \mathbf{y}_{T}=\varepsilon_{T} \text { or }|\boldsymbol{\Phi}(B)| \mathbf{y}_{T}=a d j[\mathbf{\Phi}(B)] \varepsilon_{T}
$$


where $\boldsymbol{\Phi}(B)=\mathbf{\Phi}_{0}-\boldsymbol{\Phi}_{1}(B)$. Stationarity of $\mathbf{y}_{T}$ requires the roots of $\left|\mathbf{\Phi}\left(z^{-1}\right)\right|=0$ to lie strictly inside the unit circle (see, among others, Tiao and Guttman, 1980, Osborn, 1991; Franses, 1994, 1996a; Franses and Paap, 2005; del Barrio Castro and Osborn, 2008).

As an example, consider the $\operatorname{PAR}(2 ; 4)$ model

$$
y_{t_{s}}=\phi_{1, s} y_{t_{s}-1}+\phi_{2, s} y_{t_{s}-2}+\varepsilon_{t_{s}}
$$

which can be written as

$$
\boldsymbol{\Phi}_{0} \mathbf{y}_{T}=\boldsymbol{\Phi}_{1} \mathbf{y}_{T-1}+\varepsilon_{T}
$$

for which the characteristic equation is

$$
\left|\boldsymbol{\Phi}_{0}-\mathbf{\Phi}_{1} z\right|=\left|\begin{array}{llll}
1 & 0 & -\phi_{2,1} z & -\phi_{1,1} z \\
-\phi_{1,2} & 1 & 0 & -\phi_{2,2} z \\
-\phi_{2,3} & -\phi_{1,3} & 1 & 0 \\
0 & -\phi_{2,4} & -\phi_{1,4} & 1
\end{array}\right|=0
$$

Hence, when the nonlinear parameter restriction

$$
\begin{aligned}
& \mid \phi_{2,2} \phi_{1,3} \phi_{1,4}+\phi_{2,2} \phi_{2,4}+\phi_{2,1} \phi_{1,2} \phi_{1,3}+\phi_{2,1} \phi_{2,3}+\phi_{1,1} \phi_{1,2} \phi_{1,3} \phi_{1,4} \\
& +\phi_{1,1} \phi_{1,2} \phi_{2,4}+\phi_{1,1} \phi_{1,4} \phi_{2,3}-\phi_{2,1} \phi_{2,2} \phi_{2,3} \phi_{2,4} \mid<1
\end{aligned}
$$

is imposed on the parameters, the VS representation of the $\operatorname{PAR}(2 ; 4)$ model is stationary (see Franses and Paap, 2005). When $\phi_{2, s}=0$ for all $s$, that is we have the $\operatorname{PAR}(1 ; 4)$ model, then the stationarity condition reduces to: $\left|\phi_{1,1} \phi_{1,2} \phi_{1,3} \phi_{1,4}\right|<1$, which is equivalent to our condition $\left|\xi_{t, l}\right|<1$, or in other words, that the absolute value of $|\boldsymbol{\Phi}(l)|$ is less than one. 